\title{
Can action observation modulate balance performance in healthy subjects?
}

\author{
Roberto Gatti ${ }^{1,6}$, Elisabetta Sarasso ${ }^{1,2,3^{*}}$ (D), Mattia Pelachin ${ }^{4}$, Federica Agosta ${ }^{3}$, Massimo Filippi ${ }^{3,5}$ \\ and Andrea Tettamanti ${ }^{1,2,4}$
}

\begin{abstract}
Background: Action observation activates brain motor networks and, if followed by action imitation, it facilitates motor learning and functional recovery in patients with both neurological and musculoskeletal disorders. To date, few studies suggested that action observation plus imitation can improve balance skills; however, it is still unclear whether the simple repetitive observation of challenging balance tasks is enough to modify postural control. Thus, the primary aim of this study was to investigate whether repetitive action observation of balance exercises without imitation has the potential to improve balance performance; the secondary aim was to estimate the different training effects of action observation, action observation plus imitation and balance training relative to a control condition in healthy subjects.
\end{abstract}

Methods: Seventy-nine healthy young adults were randomly assigned to 4 groups: action observation, action observation plus imitation, balance training and control. The first three groups were trained for about 30 minutes every day for three weeks, whereas the control group received no training. Center of pressure path length and sway area were evaluated on a force platform at baseline and after training using posturographic tests with eyes open and closed.

Results: As expected, both action observation plus imitation and balance training groups compared to the control group showed balance improvements, with a medium to large effect size performing balance tasks with eyes open. Action observation without imitation group showed a balance improvement with eyes open, but without a significant difference relative to the control group.

Conclusions: Both action observation plus imitation and balance training have similar effects in improving postural control in healthy young subjects. Future studies on patients with postural instability are necessary to clarify whether AOT can induce longer lasting effects. Action observation alone showed a trend toward improving postural control in healthy subjects, suggesting the possibility to study its effects in temporarily immobilized diseased subjects.

Keywords: Action observation, Action observation training, Balance, Postural control

\section{Background}

Since childhood, humans demonstrate an extraordinary ability to predict another individual's movement, to adapt their motor plans according to another person's intention and to learn from other people's behaviour [1]. The neural substrate underlying observation, understanding and imi

\footnotetext{
* Correspondence: sarasso.elisabetta@hsr.it

${ }^{1}$ Laboratory of Movement Analysis, San Raffaele Scientific Institute, via

Olgettina 58, 20132 Milan, Italy

${ }^{2}$ Degree Course in Physiotherapy, Vita-Salute San Raffaele University, Milan, Italy

Full list of author information is available at the end of the article
}

tation of movements is the mirror neuron system [2]. The visual information is primarly processed in the visual system and then projected to the mirror neuron system, which is involved in understading the meaning of actions $[3,4]$.The mirror neuron system is mainly located in the fronto-parietal regions and plays an important role in building a motor memory modulating the motor behavior of the observer $[3,4]$.

Many studies have demonstrated that the observation of meaningful actions also stimulates the activity of motor and motor-related networks without any movement execution.

(C) The Author(s). 2019 Open Access This article is distributed under the terms of the Creative Commons Attribution 4.0 International License (http://creativecommons.org/licenses/by/4.0/), which permits unrestricted use, distribution, and 
Action observation (AO) can be interpreted as a form of "motor simulation" [5] evoking an internal representation of the observed movement also called "motor resonance" [6], which involves motor brain areas similar to those usually firing during movement. As suggested by Jeannerod, mental representation of movement contains many charachteristics of the future action such as the goal, the motor program and its consequences $[5,7]$. AO does not only share a common brain pattern with motor execution but it can also facilitate movement performance. Most pieces of evidence have showed that the observation of goal-directed actions stimulates a mental re-enactement of the observed actions and facilitates action imitation [8].

In the last few years, $\mathrm{AO}$ followed by imitation of the observed exercises (AOT) has emerged as an alternative and complementary rehabilitative approach in patients with central nervous system lesions, even in the presence of poor residual movements, taking advantage of the possibility to exercise motor networks offline [8]. Several studies have demonstrated the effect of AOT in promoting motor learning and functional recovery after stroke [9], in people affected by Parkinson's disease [10, 11], in children with cerebral palsy [12], and also in post-surgical orthopedic patients [13].

Increasing attention has been addressed to the role of AOT in improving postural control [1]. In particular, three studies showed an improvement on balance performance in chronic stroke subjects after AOT of walking abilities [14-16]. A possible neurophysiological explanation could be that locomotor adaptation is modulated by observing actions of others such as walking in challenging environment: the greater the postural sway observed, the greater the postural countermeasures adopted to adapt locomotor behaviour [17]. Moreover, Bhatt and Pai suggested that the mere observation of a slippery walking can induce awareness during real walking, thus promoting a greater stability [18]. Taube et al. also investigated the effect of $\mathrm{AO}$ associated with motor imagery on balance performances [19]. In this latter study, the authors showed that both motor imagery and $\mathrm{AO}$ associated with motor imagery of postural exercises can reduce postural sway during stance with and without unexpected externally perturbations [19].The same authors showed that brain regions known to be involved in the execution of balance tasks are also active during imagination and $\mathrm{AO}$ plus imagination of balance tasks [20]. This suggests the idea that even $\mathrm{AO}$ alone can be successfully used to improve postural control and to reduce the risk of falls in temporarily immobilized patients.

In order to understand the real contribution of $\mathrm{AO}$ alone on postural stability, the main aim of the present study was to assess whether a multi-session training consisting of $\mathrm{AO}$ of balance tasks can improve postural sway. Moreover, we compared the effect of AO with other well-known training approaches such as AOT and balance training (BT). We expected subjects trained with $\mathrm{AOT}$ and $\mathrm{BT}$ to improve balance performance by reducing postural sway; we also hypothesized that $\mathrm{AO}$ could play a role in modifying postural behaviour, even if with a smaller effect size relative to AOT and BT.

\section{Methods}

\section{Participants}

Seventy-nine healthy young subjects (39 females, 40 males, mean age 21.39 years \pm SD 1.73), recruited among University students, participated in this study (Table 1). Inclusion criteria were the following: 1 ) age $<30$ years; 2 ) normal or corrected to normal vision; 3) absence of neurological and orthopedic disorders; 4 ) absence of specific balance skills due to competitive sports. The current work has been carried out in accordance with The Code of Ethics of the World Medical Association (Declaration of Helsinki) for experiments involving humans; informed consent was obtained from all subjects.

\section{Training design}

The participants were randomly assigned to 4 groups: AO (action observation without imitation), BT (balance training), AOT (action observation plus imitation), and $\mathrm{CO}$ (control group). A computerized random list generator (random.org) provided the randomization list. All groups were trained for three weeks, five days per week (Monday to Friday), except for the $\mathrm{CO}$ group which received no treatment.

A total of 30 videos of challenging balance exercises performed by professional athletes, for example walking on a balance beam with eyes closed, standing on one leg on a trampoline, standing on a wobble board (see Fig. 1 for other examples), were used for AO and AOT groups. Each video provided a single exercise in both sagittal and frontal perspectives and lasted $5 \mathrm{~min}$. For each video, two versions (with a male and a female as actors) were available and were administered in accordance with the gender of participants. AO sessions consisted of carefully watching 4 different videos, with resting periods of $4 \mathrm{~min}$ between them. AOT participants watched the same 4 videos as AO group and, after each video, they imitated the exercise for $3 \mathrm{~min}$. BT participants watched 4 different videos, showing landscapes with no motor content; after each video, they were asked to perform the same balance exercise seen by $\mathrm{AO} / \mathrm{AOT}$ groups following the verbal instructions of a physiotherapist. Each exercise was performed for $3 \mathrm{~min}$. Considering videos, exercises and rest, all the groups underwent sessions lasting $32 \mathrm{~min}$.

The same training was performed for two consecutive days, with increasing level of exercise difficulty. Each subject received individual training sessions in a quiet 
Table 1 Demographic characteristics of the participants and posturographic variables at baseline

\begin{tabular}{|c|c|c|c|c|c|}
\hline & $\mathrm{CO}$ & $\mathrm{AO}$ & BT & AOT & $p$ \\
\hline Sample size (n) & 20 & 20 & 19 & 20 & - \\
\hline Age (years) & 22.00 & $21.00^{\mathrm{a}}$ & 21.00 & 21.50 & 0.03 \\
\hline [range] & [21-28] & [20-24] & [19-26] & [19-23] & \\
\hline Weight (kg) & 66.50 & 60.00 & 67.00 & 70.00 & 0.59 \\
\hline [range] & [47-83] & [50-86] & [50-85] & [50-87] & \\
\hline Height (cm) & 175.00 & 171.00 & 173.00 & 174.00 & 0.68 \\
\hline [range] & [158-190] & [159-192] & [163-193] & [160-195] & \\
\hline BMI $\left(\mathrm{kg} / \mathrm{cm}^{2}\right)$ & 21.28 & 20.86 & 21.14 & 21.98 & 0.38 \\
\hline [range] & [18.82-24.78] & [19.05-26.54] & [18.81-24.72] & [17.30-26.26] & \\
\hline \multicolumn{6}{|l|}{ Balance measures } \\
\hline CoP Sway Path Length eyes open (mm) & $767.02 \pm 120.37$ & $818.19 \pm 99.64$ & $802.61 \pm 77.72$ & $766.08 \pm 86.32$ & 0.28 \\
\hline CoP Sway Path Length eyes closed (mm) & $1139.35 \pm 226.96$ & $1138.92 \pm 593.31$ & $1213.40 \pm 219.62$ & $1122.54 \pm 252.01$ & 0.63 \\
\hline CoP Sway Area eyes open $\left(\mathrm{mm}^{2}\right)$ & $1945.20 \pm 675.74$ & $2180.27 \pm 593.37$ & $1947.27 \pm 472.17$ & $1885.15 \pm 427.41$ & 0.36 \\
\hline CoP Sway Area eyes closed $\left(\mathrm{mm}^{2}\right)$ & $4343.04 \pm 1742.13$ & $4168.93 \pm 1404.93$ & $4783.75 \pm 1997.22$ & $4156.97 \pm 1559.61$ & 0.79 \\
\hline
\end{tabular}

Values are mean [range] or \pm standard deviation. $P$ values referred to Kruskal Wallis test. ${ }^{a}=\mathrm{AO}$ group is younger than $\mathrm{CO}$ group according to post-hoc comparisons

$A O$ action observation group, $A O T$ action observation training ( $A O$ plus imitation) group, $B T$ balance training group, $B M I$ body mass index, $C O$ control group, $C O P$ center of pressure

room. The same physiotherapist, blinded to baseline assessement, trained subjects during the exercises.

\section{Balance performance assessment}

The subjects' displacement of the center of pressure (CoP) was evaluated at baseline (T0, the day before the first training session) and at the end of the training period ( $\mathrm{T} 1$, the day after the last training session) using posturographic tests executed on a force platform (KISTLER mod. 9286A, CH). Test acquisitions were performed using a sampling frequency of $100 \mathrm{~Hz}$. Participants were instructed to stand on the force platform and to look forward in upright position with arms at their sides. The balance tests consisted in the maintenance of 4 static positions (both eyes open and eyes closed): 1) bipedal stance; 2) single right stance; 3) single left stance; 4) bipedal stance on a foam pad. Bipedal stances lasted $30 \mathrm{~s}$, whereas single leg stances lasted $15 \mathrm{~s}$. Moving arms to recover balance was not allowed. At the end of each test, the subjects were allowed to sit on a chair for about $3 \mathrm{~min}$

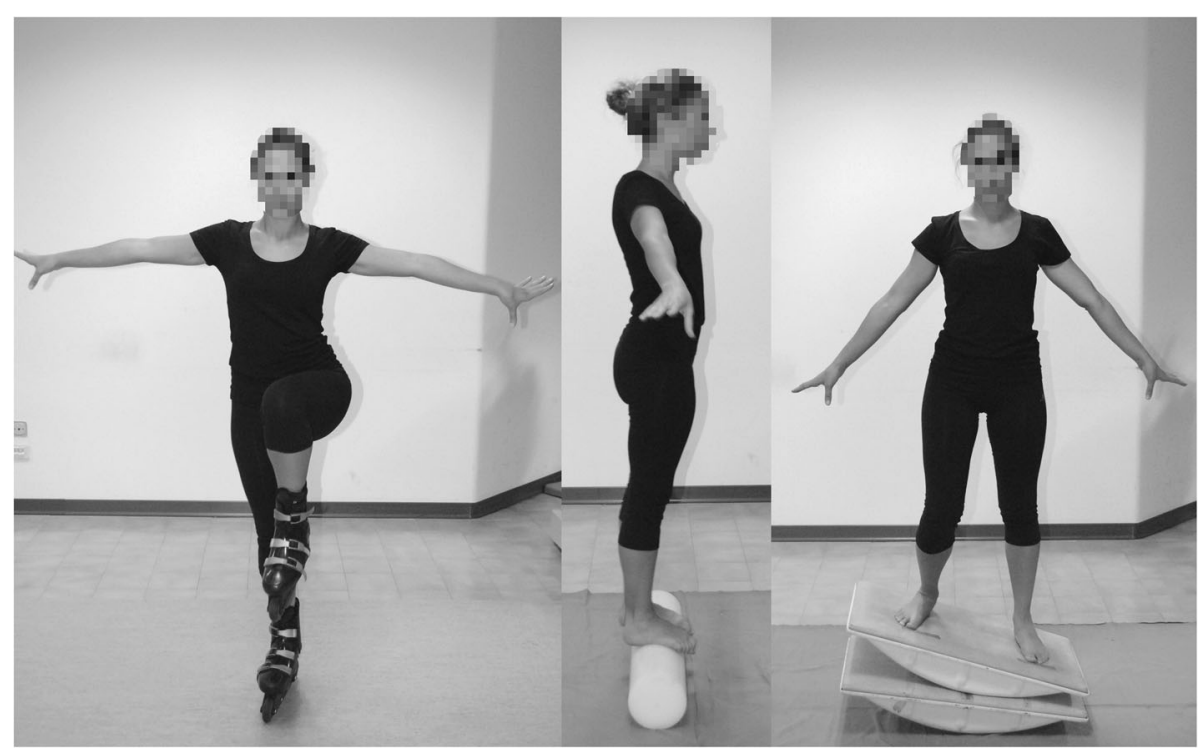

Fig. 1 The figure shows three examples of balance exercises performed by balance training (BT) and action observation training (AOT) groups 
to have a rest, and each test was repeated twice. The values of CoP oscillations (CoP Sway Path Length and Area) were analyzed using the software "Sway 1.4.1", BTS S.p.A; the best performance, defined by the lower value of CoP Sway Path Length or Area was considered.

\section{Statistical analysis}

CoP Sway Path Length and Area were expressed in $\mathrm{mm}$ and $\mathrm{mm}^{2}$ respectively. Considering the relatively small sample size and the non-normal data distribution (assessed using Kolmogorov Smirnov and Shapiro Wilk tests), non parametric statistic was used. T0 variables were compared between groups using the Kruskal Wallis test followed by Mann Whitney post-hoc comparisons. The Wilcoxon Signed Ranks test was used to assess changes overtime in each group. Differences between T0 and T1 were compared between groups using Kruskal Wallis and Mann Whitney tests. Correction for multiple comparisons was performed using Benjamini-Hochberg procedure to adjust for False Discovery Rate (alpha level $=0.05$, type I error). Statistical significance was accepted for values of $p<0.05$. The effect size (standardized mean difference) of each treatment was then calculated using Hedges' $g$ approach. Effect size was considered small if the score was 0.2 , medium if the score was around 0.5 and large with a score around or above 0.8 [21]; the effect was statistically significant if the confidence interval did not cross the zero. All data were analysed using the software SPSS 23.

\section{Results}

The groups were similar at baseline in terms of demographic variables (height, weight and BMI), except for the age that was relatively higher in $\mathrm{CO}$ group relative to AO group. Moreover, no significant differences in balance performance were found between the 4 groups at T0 (see Table 1).

\section{Within-group changes}

All the 3 trained groups showed improvements in balance performance at $\mathrm{T} 1$ relative to $\mathrm{T} 0$, while the $\mathrm{CO}$ group did not (Table 2). AO group showed a reduced CoP Sway Area during eyes open balance tasks. BT group showed a reduced CoP Sway Area during eyes open balance tasks and also a reduced CoP Sway Path Length during balance tasks both with eyes open and eyes closed. AOT group improved both CoP Sway Path Length and Area during eyes open balance tasks.

\section{Between-group changes}

The comparison of balance task changes between groups over time showed significant differences in favour of the AOT and BT groups compared to the CO group (Table 2). Specifically, the BT group showed an improved CoP Sway Path Length performing balance tasks with both eyes open and closed, while AOT group only during open eyes balance tests. Relative to the $\mathrm{CO}$ group, $\mathrm{AO}$ did not show any significant change overtime.

\section{Effect size analysis}

As illustrated in Fig. 2, we found that AOT and BT showed a significant and medium to large effect size (0.7 [0.051.35 C.I.]) by reducing CoP Sway Path Length during eyes open tasks; the AO group showed a small to medium effect size, which however was not statistically significant (0.3 [-0.32-0.93 C.I.]. Performing eyes closed balance tasks, no group achieved a significant effect, with a trend toward a significant and medium/large effect size in the BT group (0.6 [- 0.02-1.28 C.I.]. No effect was found in the control group in both balance tasks.

\section{Discussion}

This study showed that AOT and BT can modify postural control by reducing postural sway in healthy young subjects. As expected, both AOT and BT showed also a significant effect size on balance performance, with a medium to large effect performing tasks with eyes open $[16,22]$. AO without imitation showed significant reduction of CoP Sway Area during eyes open balance tasks, but without reaching a significant difference relative to the control group or a significant effect size. Certainly, AOT is expected to be more effective in improving motor performance relative to $\mathrm{AO}$ alone; however, the small effect induced by AO might become clinically important if obtained in patients with postural instability. Thus, our results suggest the possibility to study $\mathrm{AO}$ effects to train motor learning of balance tasks in temporarily immobilized diseased subjects. The mere observation of actions is actually part of the complex mechanism of motor learning: $\mathrm{AO}$ can evoke an internal representation of the observed movement $[7,23]$, strengthening the formation of a motor memory and facilitating the subsequent motor performance [1, 10]. Indeed, $\mathrm{AO}$ activates brain areas similar to those recruited performing the given action, such as the motor brain network and the mirror neuron system [6]. These areas are usually engaged during the acquisition of new motor skills and particularly during the motor learning of goal-directed behaviours [10].

Previous studies suggested that the observer is able to generate predictions about motor behaviours of others by covertly simulating the observed action [24]. Moreover, humans can easily detect movement errors and also adapt their behaviour in order to avoid the observed errors: for instance, the observation of a person that is about to fall induces a state of awareness which can reduce risk of fall when the observer walks on unstable surfaces [18]. Most recently, Tia et al. confirmed the presence of a "contagion effect" which consists of a contagious postural reaction following the observation of 


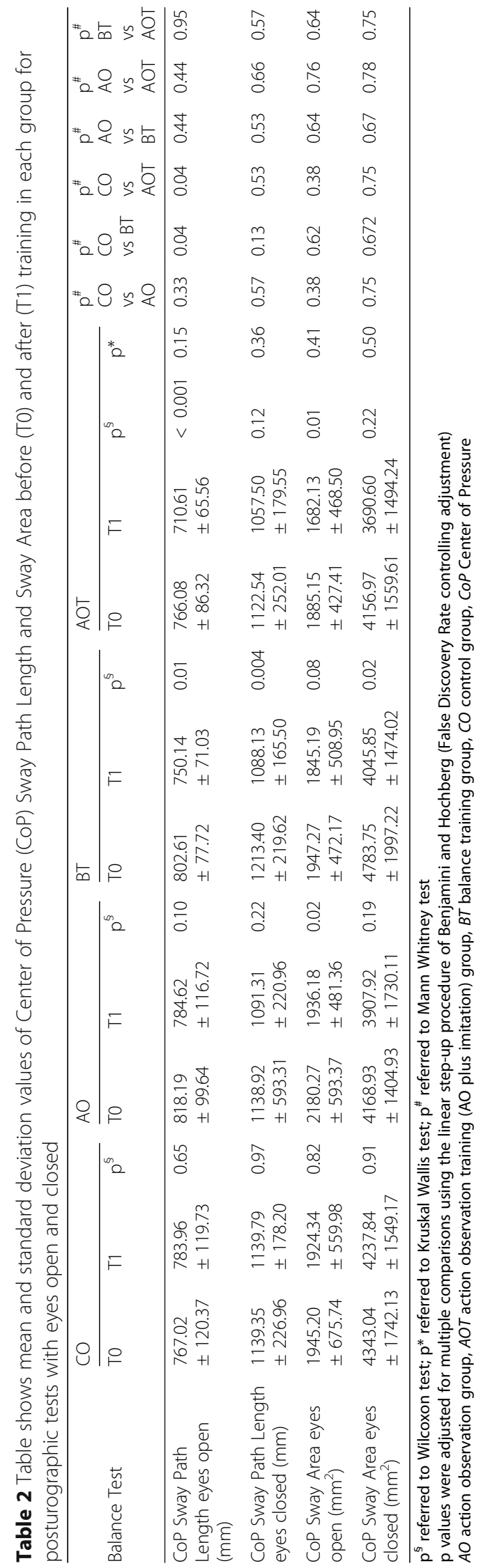




\section{Mean CoP Sway Path Length eyes open (A)}

\begin{tabular}{|c|c|c|c|c|}
\hline Group & \multicolumn{4}{|c|}{ Effect size [ $95 \%$ confidence interval] } \\
\hline \multirow{5}{*}{$\begin{array}{l}\text { Control } \\
\text { Action Observation } \\
\text { Balance Training } \\
\text { Action Observation Training }\end{array}$} & $-0.14[-0.76,0.48]$ & 1 & 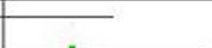 & \\
\hline & $0.30[-0.32,0.93]$ & & 1 & \\
\hline & $0.69[0.05,1.33]$ & & & \\
\hline & $0.71[0.07,1.35]$ & & & \\
\hline & -1 & $\begin{array}{l}-0.5 \\
\text { Worsening }\end{array}$ & $\begin{array}{cc}0.5 \\
\text { Improvement }\end{array}$ & 1 \\
\hline
\end{tabular}

Mean CoP Sway Path Length eyes closed (B)

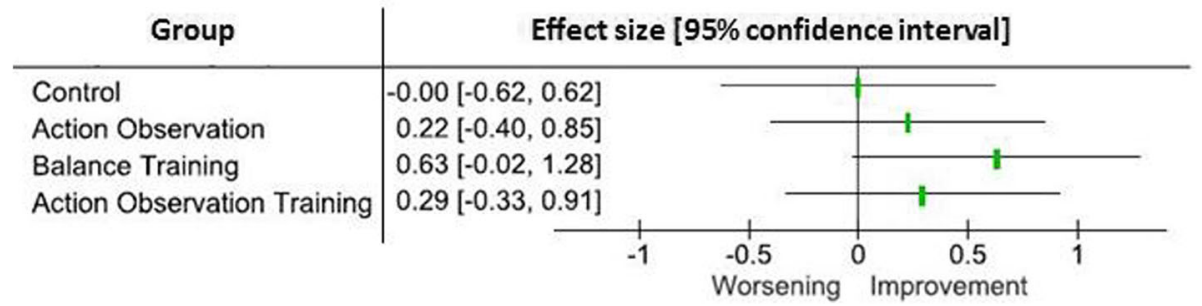

Fig. 2 The figure shows the effect size of each training on Center of Pressure (CoP) Sway Path Length during eyes open (a) and eyes closed (b) balance tests

human imbalance; interestingly authors demonstrated that the postural response can be modulated by habituation, suggesting a possible learning effect $[25,26]$. The size of the observed postural sway is also correlated with the subsequent motor response of the observer during the same balance task [17]. For this reason, a crucial issue is the difficulty level of the proposed training: not only the executed exercises must be challenging in order to obtain balance improvement, but also the observed perturbations need to be large to obtain an aftereffect in the observer [17]. Thus, we proposed videos showing complex balance exercises, in order to stimulate the attention about details of different postural reactions and balance strategies used to overcome destabilizations. Interestingly we tested subjects in different balance conditions relative to those observed, anyway obtaining an improvement of postural sway. These results suggest that the mere observation of another person in a challenging environment is enough to increase postural awareness and stimulate postural countermeasures also performing different balance tasks.

Interestingly, it would seem that subjects involved in $\mathrm{AO}$ and AOT performed better in tests with eyes open. As known, the vision is an important mechanism that works together with proprioceptive and vestibular feedbacks to maintain balance [27]; in particular, visual information is continuously integrated by the cerebellum in order to correct movement errors and detect the better timing of execution. Moreover, the visual stimulus is known to be salient in enhancing attentive functions performing actions [28], thus AO can be interpreted as an attentional strategy that could improve externally focused attention during the execution of attention-demanding tasks such as balance exercises. This point is particularly interesting, considering recent evidence showing that the risk of falls in elderly subjects is strongly correlated with the performance at cognitive tests. In particular, executive, attentive and visuospatial alterations contributed to augment risk of falls in elderly subjects together with motor difficulties such as increased body sways, low reaction time and gait speed, weak muscle strength, and poor visual contrast [29]. Thus, it would be interesting to study the possible effects of $\mathrm{AO}$ and $\mathrm{AOT}$ in stimulating cognitive networks in elderly people to improve balance and reduce risk of falls.

Moreover, recent studies suggested that also motor imagery and $\mathrm{AO}$ associated with motor imagery can improve balance performance by reducing postural sway during stance with and without perturbations [19]. These two strategies can also induce functional brain activation in regions known to be involved in the execution of balance tasks [20]. More recent evidence suggested that AO and motor imagery can be considered as different parts of the same paradigm [30] and their effects may influence each other in a very specific way [31]: motor imagery can modulate the effects of $\mathrm{AO}$ on motor learning [32] and greater brain activations in cortical-subcortical networks were found in the AO plus motor imagery condition relative to $\mathrm{AO}$ and motor imagery separately [20,33,34]. Considering these findings, future studies are needed to 
confirm the potential superiority of a combined AOTmotor imagery approach to improve postural instability.

This study is not without limitations. Firstly, we proposed balance exercises and tests involving mainly feedforward mechanisms. Further studies are needed to analyse also feedback balance strategies. Moreover, this is a pilot study, the number of subjects is relatively small and no information about the long-term effects of trainings on postural sway is available. A possible advantage of AOT is that it could potentiate motor learning providing a longer lasting effect relative to motor training alone [8]. Thus, future studies should investigate the long-term effects of this approach on balance performances. We did not find any significant difference between different trainings, but we included only healthy young subjects whithout any balance impairment. Future studies should involve a population of patients with postural instability to investigate if results reach a clinical impact. AO without imitation might be proposed in temporarely immobilized diseased patients to promote learning of motor strategies useful to perform future motor esercises. However further studies are needed to probe this hypothesis. Finally, it would be interesting to use functional magnetic resonance imaging in order to understand the neural mechanisms underlying our findings.

\section{Conclusion}

According to our findings both AOT and BT have similar effects in improving postural control in healthy young subjects, with a medium to large effect size performing balance tasks with eyes open. AO alone showed the possibility to modulate motor behaviours during balance tasks in some healthy young subjects, but without significant changes relative to the control group. Our findings also showed no significant differences between AO, AOT and BT effects on balance performance in healthy young subjects. However, further studies are needed to: i) investigate the effects of $\mathrm{AO}$ to promote learning of motor strategies useful to perform future motor esercises in temporarily immobilized diseased subjects; ii) define if AOT of balance exercise relative to BT has greater effect to improve postural control earlier and with more long-lasting results in patients with postural instability.

\section{Abbreviations}

AO: Action observation (without imitation); AOT: Action observation training (AO plus imitation); BT: Balance training; CO: Control group; CoP: Center of pressure; T0: Baseline; T1: After three weeks of training

\section{Acknowledgments}

We would like to thank Elena Paci and Eleonora Rebecchi for their contribution to this project.

Funding

No funding.

\section{Availability of data and materials}

All data generated or analysed during this study are included in this published article. Please contact author for raw data requests.

\section{Authors' contributions \\ All authors approve the submitted manuscript and they are all fully involved in the study and preparation of the manuscript. RG, AT defined the design of the work; RG, ES, MP, AT were involved in the acquisition and analysis of data. $R G$, ES wrote the manuscript. FA, MF revised the manuscript critically for important intellectual content. RG, ES, MP, FA, MF, AT gave the final approval of the version to be published.}

\section{Ethics approval and consent to participate}

The work described has been carried out in accordance with The Code of Ethics of the World Medical Association (Declaration of Helsinki) for experiments involving humans; informed consent was obtained from all subjects.

\section{Consent for publication}

Not applicable.

\section{Competing interests}

The authors declare that they have no competing interests and received no financial support.

\section{Publisher's Note}

Springer Nature remains neutral with regard to jurisdictional claims in published maps and institutional affiliations.

\section{Author details}

'Laboratory of Movement Analysis, San Raffaele Scientific Institute, via Olgettina 58, 20132 Milan, Italy. ${ }^{2}$ Degree Course in Physiotherapy, Vita-Salute San Raffaele University, Milan, Italy. ${ }^{3}$ Neuroimaging Research Unit, Institute of Experimental Neurology, Division of Neuroscience, San Raffaele Scientific Institute, Vita-Salute San Raffaele University, Via Olgettina, 60, 20132 Milan, Italy. ${ }^{4}$ Rehabilitation Department, San Raffaele Hospital, Milan, Italy.

${ }^{5}$ Department of Neurology, Institute of Experimental Neurology, Division of Neuroscience, San Raffaele Scientific Institute, Vita-Salute San Raffaele University, Milan, Italy. ${ }^{6}$ Physiotherapy Unit, Humanitas University and Humanitas Clinical and Research Center, Rozzano, Italy.

Received: 28 November 2017 Accepted: 27 December 2018

Published online: 22 January 2019

\section{References}

1. Patel M. Action observation in the modification of postural sway and gait: theory and use in rehabilitation. Gait Posture. 2017;58:115-20.

2. Rizzolatti G, Craighero L. The mirror-neuron system. Annu Rev Neurosci. 2004:27:169-92

3. Buccino $\mathrm{G}$, et al. Neural circuits underlying imitation learning of hand actions: an event-related fMRI study. Neuron. 2004;42(2):323-34.

4. Vogt $S$, et al. Prefrontal involvement in imitation learning of hand actions: effects of practice and expertise. Neuroimage. 2007;37(4):1371-83.

5. Jeannerod M. Neural simulation of action: a unifying mechanism for motor cognition. Neuroimage. 2001;14(1 Pt 2):S103-9.

6. Rizzolatti $\mathrm{G}$, Sinigaglia $\mathrm{C}$. The functional role of the parieto-frontal mirror circuit: interpretations and misinterpretations. Nat Rev Neurosci. 2010;11(4): 264-74

7. Jeannerod $M$. The origin of voluntary action: history of a physiological concept. C R Biol. 2006;329(5-6):354-62.

8. Sarasso $E$, et al. Action observation training to improve motor function recovery: a systematic review. Arch Physiother. 2015;5:14

9. Franceschini $M$, et al. Clinical relevance of action observation in upper-limb stroke rehabilitation: a possible role in recovery of functional dexterity. A randomized clinical trial. Neurorehabil Neural Repair. 2012;26(5):456-62.

10. Agosta F, et al. Brain plasticity in Parkinson's disease with freezing of gait induced by action observation training. J Neurol. 2017;264(1):88-101.

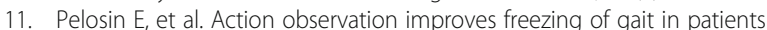
with Parkinson's disease. Neurorehabil Neural Repair. 2010;24(8):746-52. 
12. Kim JY, Kim JM, Ko EY. The effect of the action observation physical training on the upper extremity function in children with cerebral palsy. J Exerc Rehabil. 2014;10(3):176-83.

13. Bellelli $\mathrm{G}$, et al. Action observation treatment improves recovery of postsurgical orthopedic patients: evidence for a top-down effect? Arch Phys Med Rehabil. 2010;91(10):1489-94.

14. Kim JH, Lee BH. Action observation training for functional activities after stroke: a pilot randomized controlled trial. NeuroRehabilitation. 2013;33(4): 565-74.

15. Park CS, Kang KY. The effects of additional action observational training for functional electrical stimulation treatment on weight bearing, stability and gait velocity of hemiplegic patients. J Phys Ther Sci. 2013;25(9):1173-5.

16. Park EC, Hwangbo $\mathrm{G}$. The effects of action observation gait training on the static balance and walking ability of stroke patients. J Phys Ther Sci. 2015; 27(2):341-4.

17. Patel $\mathrm{M}$, et al. Locomotor adaptation is modulated by observing the actions of others. J Neurophysiol. 2015;114(3):1538-44.

18. Bhatt T, Pai YC. Can observational training substitute motor training in preventing backward balance loss after an unexpected slip during walking? J Neurophysiol. 2008;99(2):843-52

19. Taube W, et al. Non-physical practice improves task performance in an unstable, perturbed environment: motor imagery and observational balance training. Front Hum Neurosci. 2014;8:972.

20. Taube $W$, et al. Brain activity during observation and motor imagery of different balance tasks: an fMRI study. Cortex. 2015;64:102-14.

21. Durlak JA. How to select, calculate, and interpret effect sizes. J Pediatr Psychol. 2009;34(9):917-28.

22. Lesinski $\mathrm{M}$, et al. Effects of balance training on balance performance in healthy older adults: a systematic review and meta-analysis. Sports Med. 2015;45(12):1721-38.

23. O'Shea H, Moran A. Does motor simulation theory explain the cognitive mechanisms underlying motor imagery? A Critical Review. Front Hum Neurosci. 2017:11:72.

24. Wolpert DM, Diedrichsen J, Flanagan JR. Principles of sensorimotor learning. Nat Rev Neurosci. 2011;12(12):739-51.

25. Tia B, et al. Improvement of motor performance by observational training in elderly people. Neurosci Lett. 2010:480(2):138-42.

26. Tia B, et al. Does observation of postural imbalance induce a postural reaction? PLoS One. 2011;6(3):e17799.

27. Wade $M G$, Jones $G$. The role of vision and spatial orientation in the maintenance of posture. Phys Ther. 1997;77(6):619-28.

28. Sturnieks DL, George RS, Lord SR. Balance disorders in the elderly. Neurophysiol Clin. 2008;38(6):467-78.

29. Martin KL, et al. Cognitive function modifies the effect of physiologica function on the risk of multiple falls--a population-based study. J Gerontol A Biol Sci Med Sci. 2013;68(9):1091-7.

30. Caligiore $\mathrm{D}$, et al. Action observation and motor imagery for rehabilitation in Parkinson's disease: a systematic review and an integrative hypothesis. Neurosci Biobehav Rev. 2017;72:210-22.

31. Conson $\mathrm{M}$, et al. Action observation improves motor imagery: specific interactions between simulative processes. Exp Brain Res. 2009;199(1):71-81.

32. Lawrence G, Callow N, Roberts R. Watch me if you can: imagery ability moderates observational learning effectiveness. Front Hum Neurosci. 2013;7: 522.

33. Macuga KL, Frey SH. Neural representations involved in observed, imagined and imitated actions are dissociable and hierarchically organized. Neuroimage. 2012;59(3):2798-807.

34. Villiger $\mathrm{M}$, et al. Enhanced activation of motor execution networks using action observation combined with imagination of lower limb movements. PLoS One. 2013;8(8):e72403.

Ready to submit your research? Choose BMC and benefit from:

- fast, convenient online submission

- thorough peer review by experienced researchers in your field

- rapid publication on acceptance

- support for research data, including large and complex data types

- gold Open Access which fosters wider collaboration and increased citations

- maximum visibility for your research: over $100 \mathrm{M}$ website views per year

At $\mathrm{BMC}$, research is always in progress.

Learn more biomedcentral.com/submissions 